\title{
Der DEGUM-Vorstand im Interview
}

\author{
Professor Dr. med. Peter Jecker
}

Peter Jecker ist bereits seit 2015 Vorstandsmitglied und der zweite Neupräsident, der ursprünglich aus der kleinen Sektion Kopf-Hals kommt. Für die Amtszeit als DEGUM-Präsident (2018 bis 2020) sieht er große strukturelle Aufgaben auf sich und die Fachgesellschaft zukommen, die viel Arbeit, aber auch große Chancen bedeuten.

1. Welche persönlichen Ideen/Visionen haben Sie in das Ihnen derzeitig betraute Vorstandsamt als Neupräsident gebracht?

Ich wurde 2017 in das Amt des Neupräsidenten gewählt, nachdem ich zuvor 2 Jahre lang dem Vorstand als Sekretär gedient hatte. Dort gab es viel zu organisieren, da sich die DEGUM gewissermaßen in einem Umbruch befand. Außerdem wurde ich mit der Organisation der Wissenschaftsförderung betraut. Anscheinend habe ich die mir anvertrauten Aufgaben zufriedenstellend erledigt, sodass mir damals die Position des Neupräsidenten zugetraut und ich gewählt wurde. Darauf folgt dann ja die Präsidentschaft. Dabei freut es mich natürlich besonders, dass ich nach Professor Wolf Mann bereits der zweite Neupräsident der DEGUM bin, der ursprünglich aus der relativ kleinen Kopf-Hals-Sektion entstammt.

2. Welche (Ihnen wichtigen) Punkte nehmen Sie eventuell noch in Angriff bzw. konnten Sie in Ihrer jetzigen Amtszeit realisieren, bevor diese im Herbst endet?

In Kürze müssen wir im Vorstand über die diesjährige Vergabe des Wissenschaftsund Promotionspreises entscheiden. Da muss ich noch einige Dinge vorbereiten.

Insgesamt gibt es aber kaum Punkte, die allein auf den Schultern eines Vorstandsmitglieds lasten. Wichtige Entscheidungen der vergangenen 2 Jahre, wie beispielsweise der Umzug der Geschäftsstelle in Berlin, die Initiierung einer koordinierten Beteiligung der DEGUM an der Erstellung von wissenschaftlichen Leitlinien oder auch Personalentscheidungen, waren nur zügig möglich, weil die Mitstreiter im DEGUM-Vorstand alle an einem Strang gezogen haben.

\section{Wie haben Sie ihre jetzige Amtszeit erlebt? Welches Fazit ziehen sie daraus?}

Ich kann nur eine positive Bilanz ziehen. Die Arbeit im Vorstand macht nach wie vor viel Freude. So muss es aber auch sein, denn es ist ja mehr oder weniger eine Freizeitbeschäftigung und in meiner Freizeit habe ich keine Lust, mich herumzuärgern. Entscheidend sind dabei für mich die Mitarbeiter, sei es im Vorstand selbst, in der Geschäftsstelle oder in der Akademie. Und da sind wir derzeit hervorragend aufgestellt, was Kompetenz und persönlichen Einsatz des Einzelnen betrifft. Deshalb ziehe ich daraus das Fazit, dass ich mich unter den jetzigen Rahmenbedingungen gerne auch zukünftig bei der DEGUM einbringe.

\section{Welche Erfahrungen können Sie an Ihre Nachfolger weitergeben bzw. welche The- men werden Ihrer Einschätzung nach für diese am relevantesten sein?}

Das Amt des Neupräsidenten dient in erster Linie der Vorbereitung auf die Präsidentschaft. Das bedeutet, dass der Neupräsident in 2 Jahren einer Gesellschaft vorstehen wird, die von Natur aus extrem inhomogen ist. Es sind ja derart viele Fachgruppen, von denen der Ultraschall vollkommen unterschiedlich bewertet wird und die auch unterschiedlichste berufspolitische sowie wissenschaftliche Interessen verfolgen, sodass es in diesem Dschungel schnell möglich ist, die Orientierung zu verlieren. Deshalb ist der künftige Neupräsident gut beraten, sich gründlich in die Strukturen der DEGUM einzuarbeiten, denn als Präsident wird von ihm erwartet, dass er im Interesse aller Mitglieder handelt.

\section{Mit welchen Themen möchten Sie sich künftig als Präsident für die DEGUM ein- setzen?}

Die Beteiligung der DEGUM an den wissenschaftlichen Leitlinien zur Stärkung des Ultraschalls wird ein zentrales Zukunftsthema sein. Dieses Projekt wurde vom jetzigen Präsidenten PD Dr. med. Heling angestoßen, es wird uns aber in den Folgejahren sicherlich intensiv beschäftigen. Des Weite-

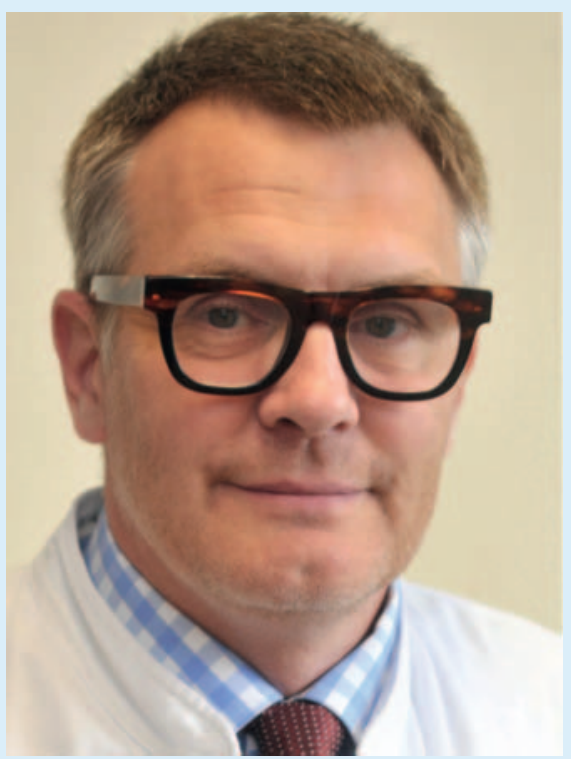

Prof. Dr. med. Peter Jecker

ren muss die Zusammenlegung der beiden Geschäftsstellen vorbereitet werden, was ja schon vor vielen Jahren beschlossen wurde. In diesem Zusammenhang sind Personalentscheidungen zu treffen, die ungeahnte Auswirkungen auf das Tagesgeschäft haben können. Das impliziert eine große Verantwortung. Und dann sind da noch zahlreiche andere Dinge, die abgearbeitet werden müssen. So sollten beispielsweise Lösungen für eine Studentenförderung durch die DEGUM entwickelt werden. Hier geht es z. B. darum zu überlegen, welche Forschungsvorhaben zukünftig gefördert werden, inwiefern die Vorstellungen von der DEGUM, der Österreichischen Gesellschaft für Ultraschall in der Medizin (ÖGUM) und der Schweizerischen Gesellschaft für Ultraschall in der Medizin (SGUM) synchronisiert werden können und so weiter. Insofern bin ich mir sicher, dass es nicht langweilig wird.

6. Worin sehen Sie aktuell die größten gesundheitspolitischen Herausforderungen für die DEGUM? Haben sich aus Ihrer Sicht seit unserem letzten Interview 2016 neue Herausforderungen ergeben?

Unser letztes Interview liegt ja noch nicht sehr lange zurück, weshalb ich sagen 
würde, dass sich da nicht viel Neues ergeben hat. Nach wie vor gilt es, den Ultraschall als Bildgebungsverfahren der ersten Wahl fest zu etablieren.

7. Nun steht das Dreiländertreffen wieder vor der Tür, bei dem traditionsgemäß ein Austausch der DEGUM-Experten mit internationalen Experten stattfindet. Wo steht die DEGUM/die Ultraschallentwicklung im internationalen Kontext? In welchen Bereichen ist Deutschland Vorreiter? Wo können wir etwas von anderen Ländern lernen?

Unter den internationalen Ultraschallgesellschaften ist meines Wissens nach die DEGUM die mitgliederstärkste Gesellschaft, auf jeden Fall in Europa. Eine Vorreiterposition sehe ich darin, wie wir in Deutschland den Ultraschall interpretieren. Da mag es die eine oder die andere Ausnahme geben, aber in der Regel besitzt der Ultraschall in unserer Vorstellung erst dann eine hohe Aussagekraft, wenn er vor dem Hintergrund des klinischen Wissens durch den behandelnden Arzt erfolgt. Und genau aus diesem Grund sollten wir von der DEGUM auch in Zukunft ganz entscheidend dafür eintreten, dass der Ultraschall in erster Linie vom Arzt persönlich durchzuführen ist und wir müssen meiner Einschätzung nach Bestrebungen aus anderen Ländern, in denen der Ultraschall - aus welchen Gründen auch immer - an medizinisches Hilfspersonal delegiert wird, entscheidend entgegentreten.

\section{Mit welchen Themen beschäftigen Sie sich derzeit im klinischen Alltag bzw. der klinischen Forschung?}

Zuerst mal habe ich eine HNO-Klinik zu leiten, in der jährlich circa 2000 Patienten stationär behandelt werden. Jeder, der eine ähnliche Tätigkeit ausübt, weiß, dass es dabei wichtig ist, klinisch immer am Ball zu bleiben, um auch in Zukunft gut aufgestellt zu sein. Was den Ultraschall betrifft, beschäftigen wir uns in erster Linie mit klinischen Fragestellungen, wie beispielsweise der Schilddrüsensonografie und der Elastografie. Gerade haben Professor Welkoborsky und ich einen neuen Kopf-Hals-Ultraschallatlas auf den Weg gebracht. Daneben muss ich mich als Ärztlicher Direktor unseres Klinikums im Alltag um Dinge wie die Krankenhaushygiene, die Umsetzung des Medizinproduktegesetzes oder auch um diverse Personalangelegenheiten und vieles mehr, gemeinsam mit der Geschäftsleitung, kümmern. Durch diese Tätigkeit bekomme ich allerdings ungeahnte Einblicke in verschiedenste Fachabteilungen und Strukturen des Hauses, was mir bei so mancher Aufgabe, die dann wieder bei der DEGUM anfällt, zugutekommt. 